\title{
Proteomic Analysis of the Cerebrospinal Fluid of Parkinson's Disease Patients Pre- and Post-Deep Brain Stimulation
}

\author{
Er-Song Wanga ${ }^{\mathrm{a}, \mathrm{b}, \mathrm{g}}$ Hui-Bin Yao ${ }^{\mathrm{a}, \mathrm{g}}$ Ying-Hui Chen ${ }^{\mathrm{c}}$ Gan Wang ${ }^{\mathrm{b}}$ Wen-Wei Gao ${ }^{\mathrm{b}}$ Yi-Rui Sun ${ }^{\mathrm{d}}$ \\ Ji-Guang Guo Jian-Wen Hu Cheng-Chuan Jiang ${ }^{\mathrm{a}, \mathrm{d}}$ Jin Hua,d

\begin{abstract}
aDepartment of Neurosurgery, Jinshan Hospital, Fudan University, Shanghai; 'bepartment of Neurosurgery, Shanghai 6th People's Hospital, Shanghai Jiaotong University, Shanghai; 'Department of Neurology, Jinshan Hospital, Fudan University, Shanghai; 'Department of Neurosurgery, Huashan Hospital, Fudan University, Shanghai, Shanghai Neurosurgical Center, Shanghai; ' Shanghai Institute

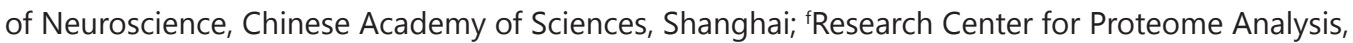
Shanghai Institutes for Biological Sciences, Chinese Academy of Sciences, Shanghai; 9These authors contributed equally to the study
\end{abstract}

\section{Key Words}

Cerebrospinal fluid • Deep brain stimulation - Parkinson's disease • Proteomics

\begin{abstract}
Aims: To investigate alterations in protein expression associated with deep brain stimulation (DBS) in an attempt to elucidate possible mechanisms of action. Methods: Cerebrospinal fluid (CSF), obtained from six Parkinson's disease (PD) patients (pre- and post-DBS) and from six normal healthy controls, was studied for differentially expressed proteins. 2-D DIGE, in combination with MALDI-TOF and TOF-TOF Mass Spectrometry (MS) or ESI-MS, was used to identify the changed proteins ( 3 PD patients and 3 controls). Selected proteins were further studied using western blotting (6 PD patients and 6 controls). Results: Twenty-one proteins were identified after MS and protein database interrogation. Apart from apolipoprotein A-I (apoA-I), the expression levels of complement C4 (C4), IgA, tetranectin, and extracellular superoxide dismutase (EC-SOD), detected by western blotting, correlated well with the 2-D DIGE results. In the follow-up period, the expression levels of C4, apoA-I and IgA were stable whereas EC-SOD and tetranectin were significantly elevated. In addition, when DBS was ceased in one patient due to a suicide attempt, the levels of EC-SOD and tetranectin significantly decreased. Conclusion: Our preliminary results suggest that variations in the expression levels of EC-SOD and tetranectin in CSF is related to DBS.
\end{abstract}




\section{Cellular Physiology and Biochemistry \\ Cell Physiol Biochem 2013;31:625-637

\begin{tabular}{|l|l|l}
\hline DOI: 10.1159/000350082 & C 2013 S. Karger AG, Basel \\
\hline
\end{tabular} \\ Publisned onilne: IVlay U6, 2013 www.karger.com/cpb \\ www.karger.com/cpb \\ Wang et}

\section{Introduction}

As one of the surgical method for Parkinson's disease (PD) therapy, deep brain stimulation (DBS) is safe, effective, adaptable, and reversible, and, since its development in 1987, is increasingly being used across the world [1-3]. Stimulation of the globus pallidus internus (GPi) or the subthalamic nucleus (STN) can reduce tremor, rigidity, bradykinesia and gait disturbances in PD patients and improve the day-to-day activities of patients with these disabling symptoms [4,5]. Although numerous studies have been performed using microdialysis, neural recording, functional imaging, and neural modeling experiments in an attempt to address the effects and mechanisms of DBS [6], its primary mechanisms of action are still poorly understood $[7,8]$.

In recent years, there has been a growing interest in applying proteomics to research disease-specific proteins in order to aid understanding of the underlying disease mechanisms [9]. These proteomic studies depend mainly on the relative amounts of protein expressed in a given population of cells in a perturbed system (i.e. a disease state or after drug treatment) compared to the unperturbed state [10].Previous studies have identified the peak at m/z 6250 that provided a satisfactory AUC value in the ROC analysis (0.956) only in discrimination of the early-stage patients with PD or MSA using CSF proteomics assay. It will be a promising application of proteomic pattern analysis to the clinical diagnosis of PD and MSA [11]. Although the exact causes of PD remain to be elucidated, a number of proteins are known to play important roles in its pathological processes [12-15], and changes in the levels of proteins in the CSF may be the result of pathological changes of the disease [9]. Moreover, the possibility of identifying and characterizing low-abundant CNS-enriched proteins and their alterations in human CSF is important for increasing the understanding of the molecular pathogenesis, and possibly also the following of therapeutic treatments, of degenerative disorders [16]. Therefore, using proteomics to investigate differentially expressed CSF proteins in a "disease state" as compared to "healthy state" can be of high value in understanding the pathogenic process of PD; similarly, investigating variations in protein expression in CSF pre-DBS (the unperturbed state) and post-DBS (the perturbed state) can be valuable in elucidating the mechanisms of action of DBS.

In this work, we have used proteomic techniques and western blotting to investigate alterations in protein expression between PD and control CSF samples and between pre-DBS and post-DBS CSF samples in an attempt to approach the mechanisms of DBS from a new perspective.

\section{Materials and Methods}

\section{Information of participants}

Six advanced idiopathic PD patients (candidates for DBS therapy; 3 males and 3 females), and six neurologically healthy, age-matched control subjects ( 3 males and 3 females) took part in the present study. The study was approved by the local ethics committee and informed consent was obtained from all participants. The PD patient selection for DBS surgery was according to the recommendations provided by Benabid [17] and Lang [18]. The main symptoms of the PD patients had initially responded to levodopa therapy, but had gradually become unresponsive to medical therapy and dopa-induced side-effects had developed (e.g. motor fluctuation, dyskinesia). All participants were in good general and mental conditions and none had undergone previous neurosurgical procedures. Participants with psychiatric disease, significant cognitive impairment, any other probable neurodegenerative disease (for PD patients)/any neurodegenerative disease (for controls), brain tumor, stroke, any inflammatory brain disease, malignant disease, systemic disorders (e.g. rheumatoid arthritis, infectious disease, diabetes mellitus, or hypertension), or who displayed abnormal laboratory test results were excluded so as not to influence the results. 


\section{Cellular Physiology and Biochemistry}

Cell Physiol Biochem 2013;31:625-637

\begin{tabular}{l|l}
\hline DOI: $10.1159 / 000350082$ & (c) 2013 S. Karger AG, Basel
\end{tabular}

Wang et al.: Proteomic Analysis of the Cerebrospinal Fluid of Parkinson's Disease Patients

\section{Surgical procedure and clinical evaluation}

After detailed explanations of the risks and potential benefits of the procedure, informed written consent was obtained from each DBS patient. Implantation of Medtronic quadripolar 3387 DBS electrodes (Medtronic Inc., Minneapolis, MN, USA) bilaterally into the STN was performed in one session under stereotaxic guidance. Intraoperative stimulation trials were carried out to determine if the stimulation improved clinical effects. The pulse generators (Kinetra, Medtronic, Minneapolis, USA) were placed under the skin in the subclavicular area in a staged procedure (one week later). Stimulation was initiated $1 \mathrm{~d}$ after pulse generator placement and electrical parameters were adjusted according to clinical improvements and side effects.

The motor scores of the Unified Parkinson's Disease Rating Scale (UPDRS) for each DBS patient were determined on the day before the operation and at 1 week, 3 months, 1 year, and 2 years after starting chronic stimulation (stimulator on) in both on-medication and off-medication states. The off-med samples were obtained after 1 hour off-medication states. Statistical analysis was performed on the UPDRS scores to measure improvements. The evaluator was blinded to the CSF results.

\section{CSF samples}

CSF specimens were obtained by lumbar puncture (LP). For each DBS patient, six consecutive CSF samples were obtained during the perioperative period on the day before DBS electrodes implantation, the day before pulse generator placement(one week after the first time CSF collection), the $7^{\text {th }}$ day after stimulation starting(one week after the second time CSF collection), and during the follow-up period at 3, 12 and 24 months after the stimulator was turned on. All LPs were performed at 7 am following fasting and $10 \mathrm{ml}$ of CSF was obtained from each LP from each participant. The cell count, glucose, total protein, and $\mathrm{pH}$ of each CSF specimen were assessed to rule out routine abnormalities. After collection, CSF samples were briefly centrifuged at $2,000 \times g$ for 10 min to pellet any cell debris, before being frozen and stored in polypropylene tubes at $-80^{\circ} \mathrm{C}$ in $1 \mathrm{ml}$ aliquots until analysis.

\section{Sample preparation for electrophoresis}

Nine CSF samples obtained during the perioperative period from three consecutive DBS patients and three CSF samples from age and sex-matched controls were selected for 2-D DIGE analysis. The depletion step was not performed, so as to avoid the loss of potential protein candidates in CSF. All selected CSF samples were precipitated with $100 \%$ ice-cold acetone in a $4: 1$ ratio of acetone to CSF at $-20^{\circ} \mathrm{C}$ for at least $4 \mathrm{~h}$ to remove salt from the sample before centrifugation at $12,000 \times g$ at $4{ }^{\circ} \mathrm{C}$ for $10 \mathrm{~min}$. The resulting pellet was washed three times with $100 \%$ ice-cold acetone and air-dried. The precipitated proteins were solubilized in a lysis buffer containing $7 \mathrm{M}$ urea, $2 \mathrm{M}$ thiourea, 4\% CHAPS, $65 \mathrm{mM}$ Tris, 2\% (v/v) protease inhibitors (protease inhibitor cocktail tablets, Roche Diagnostics, Mannheim, Germany). Protein concentrations were determined by the Bradford method with bovine serum albumin as protein standard: mean \pm SD, group A:5.32 $\pm 0.12 \mathrm{mg} / \mathrm{ml}$;group B:5.87 $\pm 0.14 \mathrm{mg} / \mathrm{ml}$; group C: $6.15 \pm 0.11 \mathrm{mg} / \mathrm{ml}$; group D: $5.68 \pm 0.09 \mathrm{mg} / \mathrm{ml}$. The potential protein losses during the acetone precipitation was about $15 \%$

\section{2-D DIGE}

CSF samples for 2-D DIGE analysis were divided into four groups with three samples per group: control group (group A), PD group (group B, for which samples were obtained 1 day before DBS electrode implantation), pre-DBS group (group C, for which samples were obtained 1 day before pulse generator placement), and the one-week group (group D, for which samples were obtained on the $7^{\text {th }}$ day after starting stimulation). There was no significant difference in total CSF protein concentration between any two groups. Samples were labeled with $N$-hydroxysuccinimidyl ester-derivatives of the cyanine dyes Cy2, Cy3, and Cy5 following the protocol of Amersham Biosciences so that $50 \mu \mathrm{g}$ of protein taken from patient and control samples was minimally labeled with $400 \mathrm{pmol}$ of Cy3 or Cy5, respectively. Cy2 was used to label the same amount of internal standard, resulting from the pooling of aliquots of all patients and controls. After incubating on ice and in the dark for $30 \mathrm{~min}$, the reaction was terminated by adding $1 \mu \mathrm{l}$ of $10 \mathrm{mM}$ lysine. The three labeled samples were then combined $(150 \mu \mathrm{g})$ and diluted with rehydration buffer (8 M urea, $2 \%$ CHAPS, $18 \mathrm{mM}$ DTT, $0.5 \%$ carrier ampholyte $\mathrm{pH} 3-10 \mathrm{NL}$ ) to $250 \mu$ l. The combined samples were loaded on a pH gradient strip (13 cm, pH 3-10, nonlinear) for isoelectric focusing (IEF) on an Ettan IPGphor system (Amersham Biosciences, Uppsala, Sweden). After active rehydration for $12 \mathrm{~h}$ at $30 \mathrm{~V}$ at $20^{\circ} \mathrm{C}$, IEF was 


\section{Cellular Physiology $\quad$ Cell Physiol Biochem 2013;31:625-637

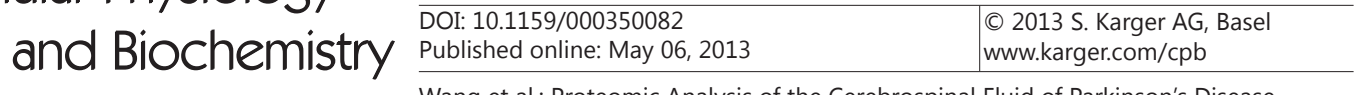 \\ Wang et al.: Proteomic Analysis of the Cerebrospinal Fluid of Parkinson's Disease Patients}

performed as follows: $500 \mathrm{~V}$ for $1 \mathrm{~h}$; $1000 \mathrm{~V}$ for $1 \mathrm{~h}$; $8000 \mathrm{~V}$ for $8 \mathrm{~h}$; and 500V for $4 \mathrm{~h}$. After IEF, the strips were first equilibrated in equilibration solution consisting of $50 \mathrm{mM}$ Tris- $\mathrm{HCl}(\mathrm{pH} 8.8), 6 \mathrm{M}$ urea, $30 \%$ (v/v) glycerol, 2\% (w/v) SDS, traces of bromophenol blue, and 1\% (w/v) DTT for $15 \mathrm{~min}$ and subsequently in the same solution except that DTT was replaced by $4 \%(\mathrm{w} / \mathrm{v})$ iodoacetamide for a further $15 \mathrm{~min}$. Equilibrated IPG strips were transferred onto $12.5 \%$ vertical polyacrylamide gels cast in low-fluorescence glass plates using a Hofer SE 600 system (GE Healthcare, Uppsala, Sweden). Electrophoresis was conducted at $1.5 \mathrm{~W} /$ gel for $20 \mathrm{~min}$ and at $3 \mathrm{~W} /$ gel constant current overnight until the bromophenol blue front reached the bottom of the gels. The Cy2-, Cy3-, and Cy5-labeled images were acquired on a Typhoon 9400 scanner (Amersham Biosciences) at the excitation/emission values of 488/520 nm, 532/580 nm, and 633/670 nm, respectively.

Intragel spot detection and quantification and intergel matching and quantification were performed automatically using the Differential In-gel Analysis (DIA) and Biological Variation Analysis (BVA) modules of DeCyder software version 6.5 (Amersham Biosciences). The gel with the highest spot count was considered the master gel. Using DIA, the Cy2, Cy3, and Cy5 images for each gel were merged, spot boundaries were automatically detected, and normalized spot volumes (protein abundance) were calculated. During spot detection, the estimated number of spots was set at 2000, and the exclude filter was set as follows: slope $>1.1$; area $<100$; peak height $<100$; and volume $<1000$. The resulting spot maps were exported to the BVA module. Gel-to-gel matching of the standard spot maps from each gel, followed by statistical analysis of protein abundance change between samples, was performed in the BVA module. Selection criteria for the detection of significantly changed protein spots were that protein spots presented at least in four of the six analyzed gels (in 12 of 18 analyzed images) and that the standardized average spot volume ratios exceed 1.5 with statistical significance (Student's $t$-test, $P<0.05$ ). In the case of 3 or more sample groups, one-way ANOVA at $95 \%$ statistical confidence (significance level, $P<0.05$ ) was set. False discovery rate(FDR) correlations [19] were applied according to the DeCyder manual.

\section{Protein digestion and mass spectrometry}

For mass spectrometric analysis, differentially expressed protein spots of interest were excised from the gel with the Ettan spot picker (Amersham Biosciences-GE Healthcare) and subjected to in-gel digestion with trypsin. Briefly, gel plugs were destained with $30 \%$ acetonitrile (ACN) in $100 \mathrm{mM}$ ammonium bicarbonate $\left(\mathrm{NH}_{4} \mathrm{HCO}_{3}\right)$ for $20 \mathrm{~min}$ and vacuum dried. Digestion buffer $\left(20 \mathrm{ng} / \mu \mathrm{l}\right.$ trypsin in $\left.20 \mathrm{mM} \mathrm{NH}_{4} \mathrm{HCO}_{3}\right)$ was then added and the samples were digested at room temperature overnight. Peptides were extracted twice with a solution containing $60 \%$ ACN and $0.1 \%$ trifluoroacetic acid (TFA). The extracted peptides were removed, dried and re-suspended in 50\% ACN and 0.1\% TFA. An equal volume of sample and $\alpha$-HCCA matrix (5 mg/ $\mathrm{ml}$ ) were spotted and mixed on the MALDI-TOF target plate. Peptide mixtures were analyzed with a Bruker Daltonics AutoFlex TOF-TOF LIFT Mass Spectrometer (Bruker Daltonics, Bremen, Germany) in positive ion reflector mode. The accelerating potential was $20 \mathrm{kV}$ with eight-shots per second. Each spectrum was internally calibrated using trypsin autolysis peaks ( $\mathrm{m} / \mathrm{z} 842.51$ or 2211.10$)$. The obtained peptide mass fingerprints spectra were analyzed by searching the non-redundant protein database National Centre for Biotechnology Information (NCBInr 20070810; 5366160 sequences, 1855569248 residues, and 193932 sequences after taxonomy filter). Search parameters were set as follows: taxonomy: Homo sapiens; enzyme: trypsin; peptide mass tolerance: $\pm 100 \mathrm{ppm}$; fragment mass tolerance: $\pm 0.8 \mathrm{Da}$; peptide charge state: $1+$; fixed modification: carbamidomethyl cysteine; maximum missed cleavages: 1 . In all protein identifications, the probability scores were greater than the score fixed as significant with a $P$ value less than 0.05 .

Samples for which unambiguous protein identity was not provided by MALDI-MS were further investigated through ESI-MS. RP-HPLC was performed using an Ettan ${ }^{\mathrm{TM}}$ MDLC (GE Healthcare) using a C18 column (0.15 mm $\times 150 \mathrm{~mm}$; Agilent Technologies, Palo Alto, CA, USA ) at a flow rate of $10 \mu \mathrm{l} / \mathrm{min}$. The mobile phase conditions consisted of: Buffer A, $0.1 \%$ formic acid in water; and Buffer B, $0.1 \%$ formic acid in ACN. The peptides were sequentially eluted with a gradient of 4 to $100 \%$ Buffer B over 60 min. The mass spectral data were acquired on an ESI-linear ion trap mass spectrometer (LTQ, Thermo Finnigan, San Jose, CA) equipped with an electrospray interface and operated in positive ion mode. The temperature of the heated capillary was set at $170^{\circ} \mathrm{C}$. A voltage of $3.3 \mathrm{kV}$ applied to the ESI needle resulted in a distinct signal. Collision energy was automatically set by the system. After acquisition of a full scan mass spectrum, ten MS/MS scans were acquired for the next ten most intense ions using dynamic exclusion. The acquired MS/ MS spectra were automatically searched against a human protein database (ipi HUMAN v3.26) using the TurboSEQUEST program in the BioWorks ${ }^{\mathrm{TM}} 3.1$ software suite. An accepted SEQUEST result had to have 


\section{Cellular Physiology and Biochemistry}

\begin{tabular}{|c|c|c|c|c|c|c|c|c|c|c|}
\hline \multirow{3}{*}{$\begin{array}{c}\text { Case } \\
\text { No. }\end{array}$} & \multirow[t]{3}{*}{ Sex } & \multirow{3}{*}{$\begin{array}{l}\text { Age } \\
\text { (year } \\
\text { s) }\end{array}$} & \multirow{3}{*}{$\begin{array}{c}\text { Duration } \\
\text { of PD } \\
\text { (years) }\end{array}$} & \multirow{3}{*}{$\begin{array}{l}\text { Medicatio } \\
\text { n Phase }\end{array}$} & \multirow{3}{*}{$\begin{array}{l}\text { H\&Y } \\
\text { Stage }\end{array}$} & \multicolumn{5}{|c|}{ UPDRS Score (part II+III) } \\
\hline & & & & & & \multirow{2}{*}{$\begin{array}{l}\text { Pre- } \\
\text { DBS }\end{array}$} & \multicolumn{4}{|c|}{ Post-DBS (\%-age improvement) } \\
\hline & & & & & & & 1 week & 3 months & 1 year & 2 years \\
\hline \multirow[t]{2}{*}{1} & M & 64 & 14 & On & III & 57 & $29(49.1)$ & $27(52.6)$ & $25(56.1)$ & $25(56.1)$ \\
\hline & & & & Off & IV & 75 & $34(54.7)$ & $30(60)$ & $29(61.3)$ & $25(66.7)$ \\
\hline \multirow[t]{2}{*}{2} & M & 59 & 8 & On & II & 28 & $21(25)$ & $16(42.9)$ & $14(50)$ & $14(50)$ \\
\hline & & & & Off & III & 59 & $31(47.5)$ & 27 (54.2) & $23(61)$ & $22(62.7)$ \\
\hline \multirow[t]{2}{*}{3} & $\mathrm{~F}$ & 61 & 10 & On & III & 33 & $23(30.3)$ & $19(42.4)$ & $18(45.5)$ & 17 (48.5) \\
\hline & & & & Off & IV & 68 & $38(44.1)$ & $30(55.9)$ & $28(58.8)$ & $28(58.8)$ \\
\hline \multirow[t]{2}{*}{4} & $\mathrm{~F}$ & 63 & 9 & On & II & 25 & $18(28)$ & $15(40)$ & $13(48)$ & $12(52)$ \\
\hline & & & & Off & III & 56 & $22(60.7)$ & $20(64.3)$ & $20(64.3)$ & $20(64.3)$ \\
\hline \multirow[t]{2}{*}{5} & $\mathrm{~F}$ & 65 & 16 & On & III & 51 & $26(49)$ & $23(54.9)$ & $23(54.9)$ & $22(56.9)$ \\
\hline & & & & Off & IV & 70 & 30 (57.1) & $26(62.9)$ & $25(64.3)$ & $25(64.3)$ \\
\hline \multirow[t]{2}{*}{$6 \#$} & M & 65 & 9 & On & III & 39 & $21(46.2)$ & $20(48.7)$ & 39\# & $40 \#$ \\
\hline & & & & Off & IV & 78 & 35 (55.1) & $30(61.5)$ & 75\# & $80 \#$ \\
\hline
\end{tabular}

Table 1. Effect of bilateral STN DBS on UPDRS motor scores. \#: Patient discontinued DBS therapy 8 months after surgery due to a suicide attempt. H\&Y, Hoehn and Yahr.

a $\Delta \mathrm{Cn}$ score of at least 0.1 (regardless of charge state). The protein identification criteria used here were based on Delta $\mathrm{CN}(\geq 0.1)$ and Xcorr (one charge $\geq 1$.9; two charges $\geq 2.2$; three charges $\geq 3.75$ ).

\section{Western blotting analysis}

All CSF samples were divided into seven groups with six samples per group, according to the time point at which the PD CSF samples were obtained: control group (group A), PD group (group B), pre-DBS group (group C), one-week group (group D), 3-months group (group E), 12-months group (group F) and 24-months group (group G). All CSF samples were used to confirm the selected data of proteomic analysis and were concentrated using ultrafiltration. Protein concentrations were determined by the Bradford method before samples were boiled in the loading buffer (60 mM Tris-HCl, pH 6.8, 25\% glycerol, 2\% SDS, $14.4 \mathrm{mM} 2$-mercaptoethanol) for $5 \mathrm{~min}$. There was no significant difference in total CSF protein content among groups. Fifty microgram protein of each sample was separated in 12.5\% SDS-polyacrylamide gels and electrotransferred to a nitrocellulose membrane (Amersham). After blocking and rinsing, blots were incubated overnight at $4{ }^{\circ} \mathrm{C}$ in one of the following primary antibodies: rabbit anti-apolipoprotein A-I polyclonal antibody ( $1: 1000)$, chicken anti-human IgA polyclonal antibody (1:3000), mouse anti-human superoxide dismutase 3 monoclonal antibody (1:500), goat anti-human C4 antibody (1:500), and mouse anti-human tetranectin monoclonal antibody (1:500). All primary antibodies were purchased from Abcam (Cambridge, MA, USA) with the exception of anti-human C4 antibody (Calbiochem, San Diego, CA, USA). Immunoblots were probed with the respective horseradish peroxidase-conjugated secondary antibodies (1:5000; Invitrogen, Carlsbad, CA, USA) and detected using SuperSignal West Pico Chemiluminescent substrate (Pierce, Rockford, IL, USA). Each immunoblot was run in triplicate or more. Detection of $\alpha$-tubulin was conducted to confirm equal loading of samples and for normalization purposes. Bands were digitized and optical densities were analyzed using ImageMaster 2D Platinum (v.5.0, Amersham Biosciences). The densities of the protein bands were normalized to that of the $\alpha$-tubulin bands.

\section{Statistical analysis}

Statistical analysis was carried out using GraphPad Prism v4.0 statistical software (GraphPad Software Inc., San Diego, CA, USA). The data were subjected to either $t$-test or one-way ANOVA and differences were considered significant when $P$ values were $<0.05$. To determine the biological coefficients of variation $(\mathrm{CVi})$, spot volumes of all analyzed protein spots were exported from DeCyder. The CVi were calculated from the SA(standardized abundance)-values of protein spots across different sets of gels. The median of CVi values per used gel set yields the biological variation [20].

\section{Results}

No significant perioperative complications occurred in any of the six DBS patients. The UPDRS motor scores significantly improved $(P<0.05)$ after DBS in both on-medication and off-medication states, and DBS resulted in more significant therapeutic effects in the off- 


\section{Cellular Physiology \\ Cell Physiol Biochem 2013;31:625-637 \\ and Biochemistry

\begin{tabular}{l|l}
\hline DOI: 10.1159/000350082 & (C) 2013 S. Karger AG, Basel
\end{tabular} \\ www.karger.com/cpb}

Wang et al.: Proteomic Analysis of the Cerebrospinal Fluid of Parkinson's Disease Patients

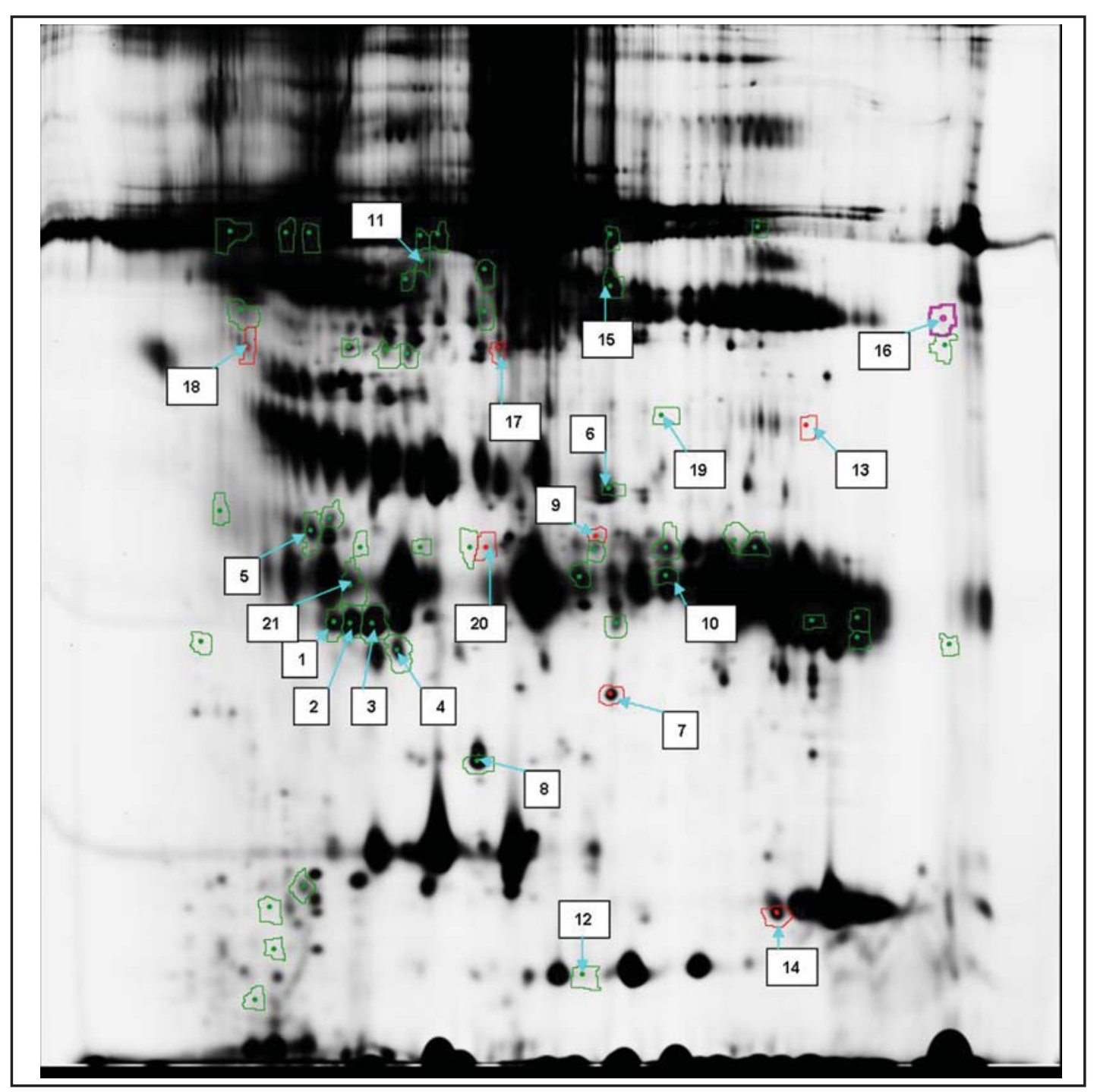

Fig. 1. Differentiated protein spots between the PD and control groups in the master gel image (Cy5-labeled) of CSF. Spots in the image were analyzed by DeCyder software and only spots with differences in standardized average spot volume ratios $>1.5$ and a $t$-test $P$ value $<0.05$ are shown (marked in green circles). Spots that were not altered significantly between the PD and control groups but analyzed by mass spectrometry are marked in red circles. Only spots which were successfully identified after mass spectrometry and protein database interrogation are manually numbered.

medication condition and 12-24 months after surgery (Table 1). All the patients selected for our study were treated with L-DOPA in the dosage of $1500 \mathrm{mg} 2000 \mathrm{mg}$ per day before their admission for DBS therapy, and keep this dosage till one week after DBS therapy (mean \pm SD $1792 \pm 246 \mathrm{mg} /$ day). The dose of L-DOPA was gradually reduced after one week. Three months after the DBS therapy, it was decreased to $750 \mathrm{mg} \sim 1250 \mathrm{mg}$ in all the patients (mean \pm SD: $1023 \pm 152 \mathrm{mg}$ /day). The mean daily antiparkinsonian treatment dose was decreased by $42 \%$ on average. Since then, the dosage was not changed in all patients during the follow-up period.

One patient discontinued the DBS therapy 8 months after surgery because of a suicide attempt. The electrodes were not removed during the follow-up period and both the suicidal thoughts and the therapeutic effect of DBS dissipated after discontinuation of the DBS therapy (Table 1). For this patient, the L-DOPA was returned to the original dosage. 


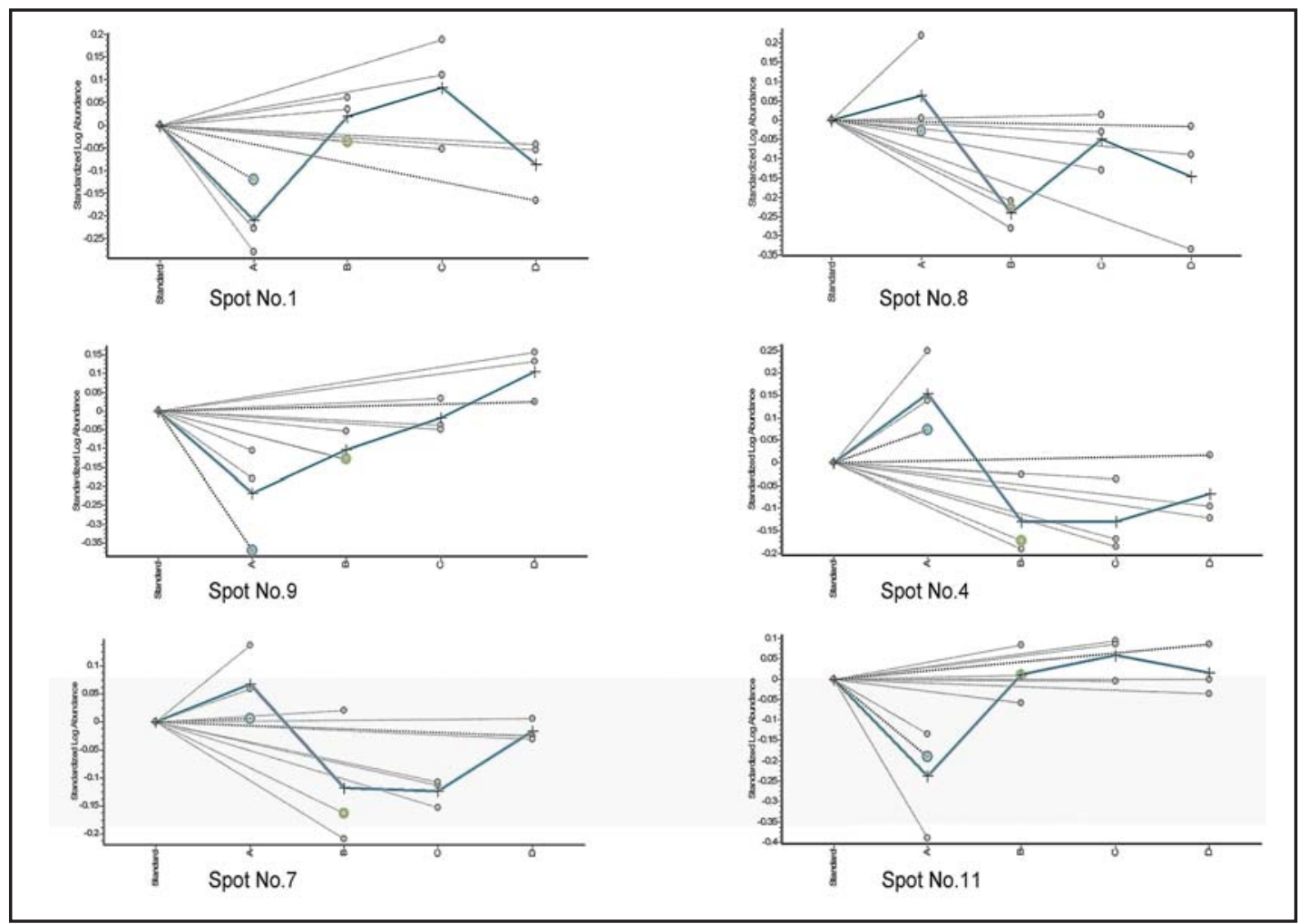

Fig. 2. Representative graph view of protein spots detected by 2-D DIGE. The volume of each protein spot was calculated automatically by the DeCyder software by displaying the abundance values relative to the pooled standard. Each dot on the graph represents the same matched spot from a different gel. Among these protein spots, spots $1,8,4$, and 11 were significantly changed between the PD and control groups, and spot 8 was also significantly changed between the PD and pre-DBS groups. Spot 9 was markedly up-regulated in the one-week group as compared to the pre-DBS group. By MS, spots 1 and 8 were identified as apoA-I, spot 9 was identified as EC-SOD, spot 4 was identified as tetranectin, spot 7 was identified as C4, and spot 11 was identified as an IgA fragment. Standard: internal standard; A: control group; B: PD group; C: pre-DBS group; D: one-week group; +: mean volume of each group.

A total of 1897 spots were detected on the master gel after analysis in the DeCyder DIA module. In order to detect as many changed proteins in the CSF as possible, we screened those statistically significant differentially expressed proteins among the A-B-C-D groups (A-B-C-D is a comparison between Group A and Group B-C-D. Group B, C, and D are all patient samples. Group A is normal control, which usually has different protein expression compared with B,C,D) and between the control and PD (A and B) groups, PD and pre-DBS (B and C) groups, as well as the pre-DBS and post-DBS one-week ( $C$ and D) groups.When applying the one-way ANOVA, protein spots were present in significantly different amounts $(p<0.05)$ and present in each and every gel. $70,48,15$, and 12 protein spots, respectively (Fig. 1)were significant when applying the FDR correlation. The average biological variation within group was 26.8\%(CVbiol:21.4-30.6\%).

Graphs of standardized abundance for a differentiated protein spot can be generated automatically by the DeCyder software by displaying the abundance values relative to the pooled standard (Fig. 2).Within these significantly altered protein spots from the A-B, B-C, and C-D groups, no identical protein spot was found in two paired groups except for one spot (spot No. 8), which emerged simultaneously in the paired PD/control (A-B) groups and PD/ pre-DBS (B-C) groups (Fig. 2).

Of these differentially expressed spots, 25 spots from A-B-C-D groups comparison were present in all analyzed images. These spots were selected and subjected to mass spectrometry 


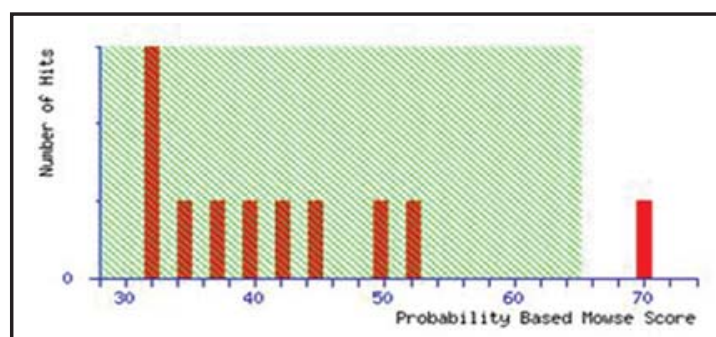

Spot No. 1

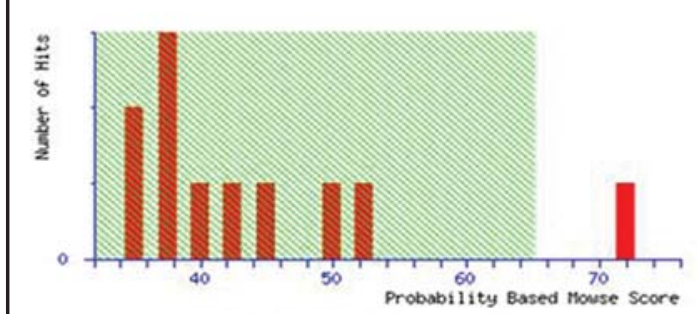

Spot No. 9

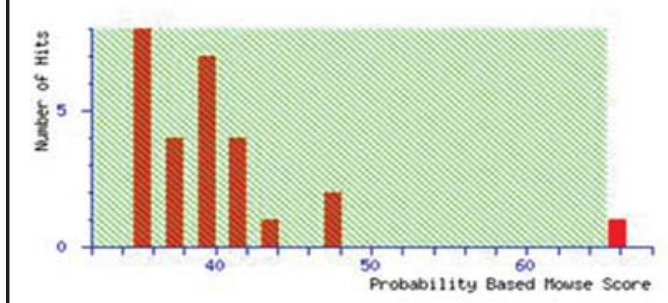

Spot No. 7

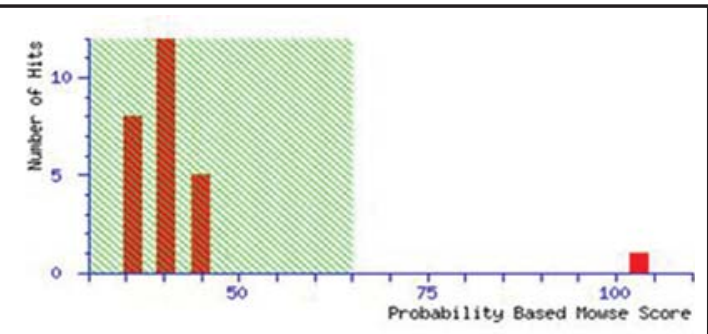

Spot No. 8

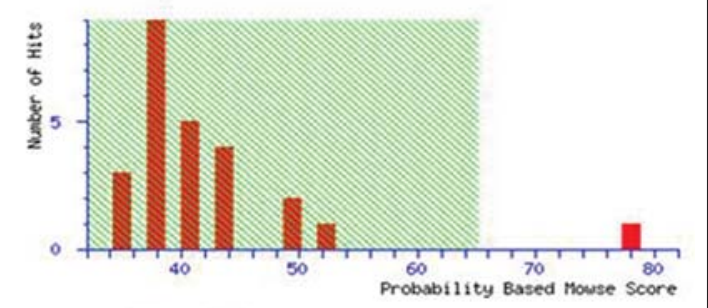

Spot No. 4

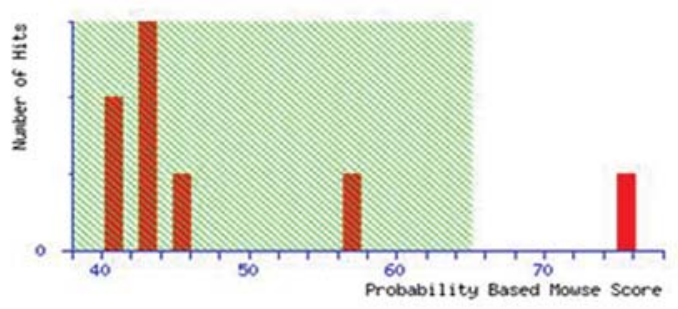

Spot No. 11

Fig. 3. MASCOT search results and probability plots corresponding to apoA-I (spots 1 and 8), EC-SOD (spot 9), tetranectin (spot 4), C4 (spot 7), and IgA (spot 11). The $m / z$-values obtained for each protein spot were used to identify the protein by searching in protein databases. Probability-based MOWSE scores were estimated as ion scores $-10 * \log _{10}(P)$, where $P$ is the probability. Individual ion scores $>65$ were considered to be significant $(P<0.05)$. High probability scores were used to identify proteins.

(MALDI-TOF or ESI) with a total of 21 proteins successfully identified. The MASCOT search engine of the MALDI-TOF instrument, which is based on the NCBI protein database, was used to identify peptide mass fingerprints and individual ion scores $>65$ were considered to be significant (Fig. 3). These identified proteins were further confirmed in the SwissProt/ TrEMBL protein database. Of these 21 proteins, 13 were significantly altered in the PD group (group B) as compared with the control group (group A), which can be regarded as PDrelated proteins; 2 were significantly altered in the pre-DBS group as compared with the PD group (spots No. 8 and 14); 3 were significantly altered in the post-DBS one-week group as compared with pre-DBS group (spots No. 9, 16 and 17); all proteins were significantly altered in general group comparisons (A-B-C-D groups). The detailed information of identified proteins is presented in Table 2 .

To confirm the proteomic analysis findings, and also to determine the exact changes of these proteins in the follow-up period, five proteins were selected for western blotting analysis. The relative intensity data had been normalized for tubulin. Normalization against tubulin performed on each individual gel.The results showed that protein bands for apoA-I, tetranectin, C4, IgA, and EC-SOD were clearly observed in all CSF samples. Semi-quantitative densitometric evaluation revealed that the expression levels of C4, IgA, tetranectin, and ECSOD correlated well with the 2-D DIGE results while the expression levels of apoA-I partially differed from the 2-D DIGE results. Moreover, the expression levels of C4, apoA-I and IgA 

Cellular Physiology $\quad$ Cell Physiol Biochem 2013;31:625-637
and Biochemistry \begin{tabular}{l|l} 
DOI: 10.1159/000350082 \\
Published onine: Tray vo, 2013 & $\begin{array}{l}\text { O 2013 S. Karger AG, Basel } \\
\text { www.karger.com/cpb }\end{array}$
\end{tabular}
Wang et al.: Proteomic Analysis of the Cerebrospinal Fluid of Parkinson's Disease Patients
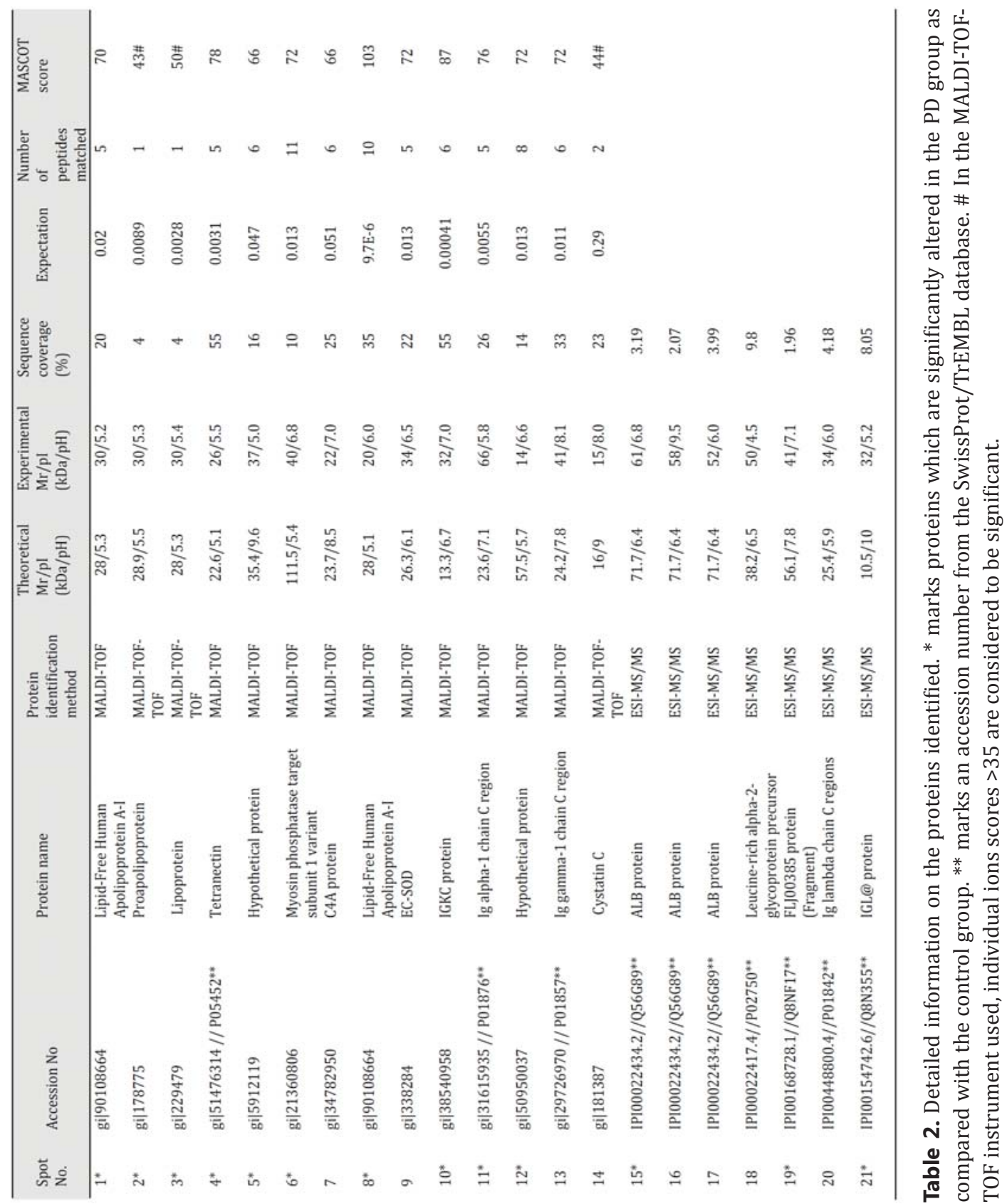

were stable in the follow-up period whereas EC-SOD and tetranectin significantly increased (Fig. 4). It is interesting to note that when the DBS was ceased due to a suicide attempt the levels of EC-SOD and tetranectin significantly decreased in parallel with a disappearance of the therapeutic effects of DBS.

\section{Discussion}

In the present study, we used 2-D DIGE, in which protein expression differences can be detected and identified with an increased confidence in comparison with the 2-DE technique [21], to analyze the changes of all proteins in the CSF of PD patients. Our data demonstrated that the significantly altered protein spots in any two paired groups (control versus PD 
Wang et al.: Proteomic Analysis of the Cerebrospinal Fluid of Parkinson's Disease Patients

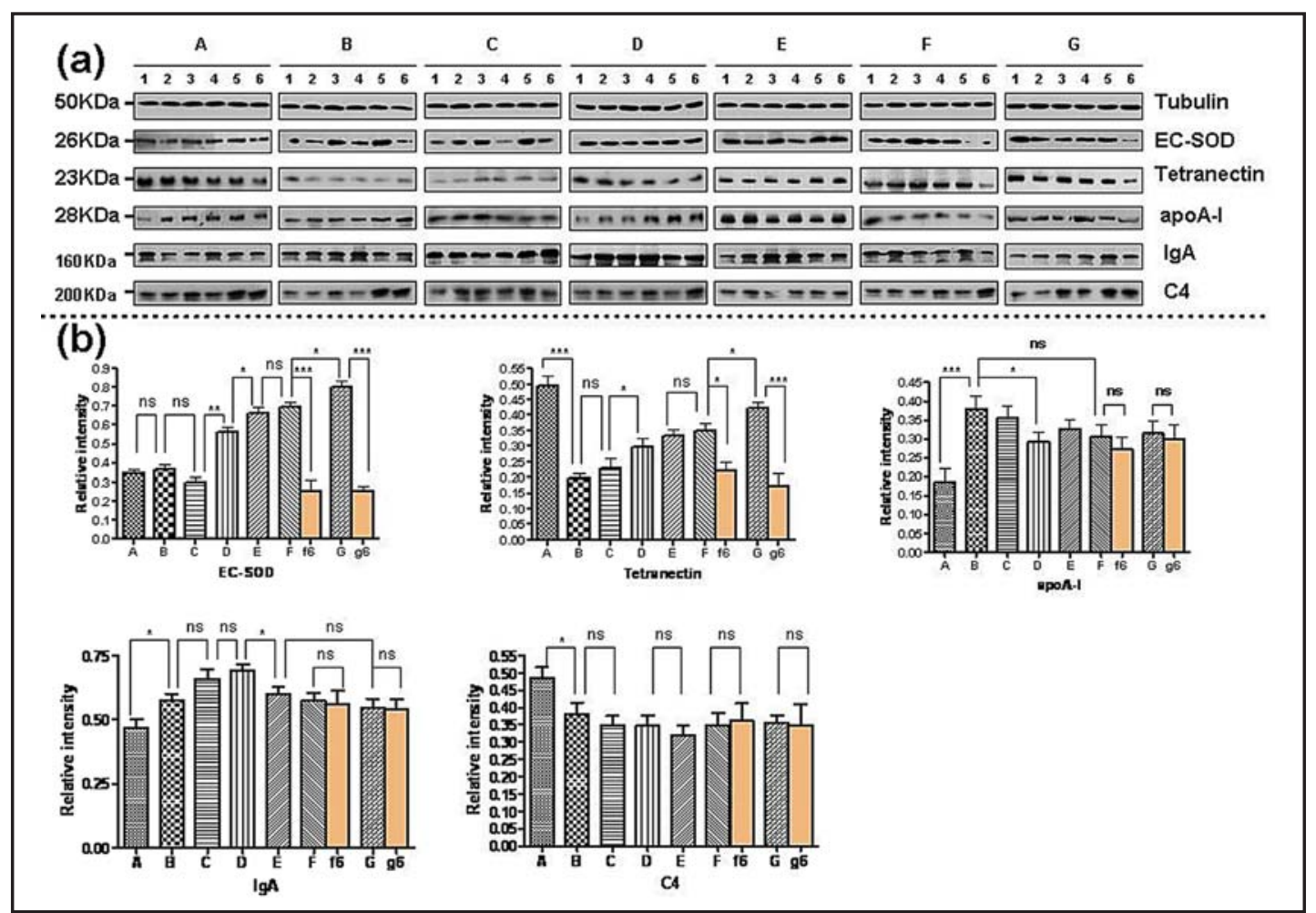

Fig. 4. Western blotting analysis for the expression of selected proteins in the CSF of PD patients and normal control subjects. (a) Representative bands of EC-SOD, tetranectin, apoA-I, IgA and C4 in the CSF of individual participants. Each lane contained $50 \mu \mathrm{g}$ of protein and each experiment was run in triplicate. The column height represents the averaged relative intensity of three times repetitional experiments of all individual samples in each group and the error bar represents standard (in group F and group G, data from the patient who ceased the DBS therapy was demonstrated exclusively as $\mathrm{f}$ and $\mathrm{g}$ ). Tubulin was detected for normalization. (b) Semi-quantitative densitometric analysis of immunoblotting bands of EC-SOD, tetranectin, apoA-I, IgA and C4. The relative intensity of the band was quantified and normalized to tubulin. A: control group; B: PD group; C: pre-DBS group; D: one-week group; E: 3-month group; F/f: 12-month group; G/g: 24-month group; f6/g6: the patient who ceased DBS therapy because of a suicide attempt. Statistical differences are indicated: ns, not significant, ${ }^{*} P<0.05,{ }^{* *} P<0.01,{ }^{* * *} P<0.001$.

groups, PD versus pre-DBS groups, and pre-DBS versus post-DBS one-week groups) were almost completely different. The possible reasons for this difference might be that: (1) the effects of STN DBS on proteins are different from that of lead insertion; (2) there are mixed effects of both lead insertion and DBS in the perioperative time, and; (3) DBS associated proteins are different from PD-related proteins.

After mass spectrometry and protein database interrogation, our data demonstrated that a total of 21 proteins were identified in the CSF. In previous CSF proteomics in PD studies, several differentially expressed proteins such as apoA-I, complement C4, and Cystatin C were identified [22, 23]. Some of these 21 proteins were identified for the first time in this work.

In order to confirm the results of the 2-D DIGE analysis, and also to know the exact changes of these proteins in the follow-up period, we further performed western blotting analysis on all collected CSF samples by using specific antibodies against apoA-I, tetranectin, C4, IgA and EC-SOD. The 2-D DIGE protein results were largely confirmed by western blotting analysis, except that of apoA-I. This discrepancy is possibly due to the fact that the antibody used for apoA-I is not isoform specific and that western blotting gives only information on total apoA-I immunoreactivity. Data from western blotting analysis revealed a significant increase in EC-SOD and tetranectin levels in the CSF of PD patients during the follow-up 


\section{Cellular Physiology and Biochemistry}

Cell Physiol Biochem 2013;31:625-637

\begin{tabular}{|l|l|}
\hline DOI: 10.1159/000350082 & C 2013 S. Karger AG, Basel \\
\hline
\end{tabular}

Wang et al.: Proteomic Analysis of the Cerebrospinal Fluid of Parkinson's Disease Patients

period, whereas the expression levels of $\mathrm{C} 4$, apoA-I and IgA were very stable. The changes in EC-SOD and tetranectin paralleled the observed improvement in parkinsonian signs and symptoms and it is of interest to note that the levels of EC-SOD and tetranectin significantly decreased upon withdrawal of DBS stimulation. These results suggest that the differences in the expression levels of EC-SOD and tetranectin are related to the STN DBS. Therefore, EC-SOD and tetranectin may serve as potential biomarkers in PD patients. Certainly, these preliminary findings need to be confirmed with a larger study.

EC-SOD is found in the extracellular matrix of tissues and functions to prevent cell and tissue damage caused by extracellularly produced reactive oxygen species (ROS) [24]. Although the physiological roles of this enzyme in the brain are not completely understood, it is primarily localized in the mouse brain to the hippocampus, striatum, suprachiasmatic nuclei and the habenula [25]. In the present study, bilateral STN DBS induced an increased expression of EC-SOD in PD patients.

To date, the biological function of tetranectin has not been fully elucidated [26]. One previous study indicated that tetranectin was involved in the degradation of extracellular matrix proteins in the brain [27]. Immunohistochemistry showed tetranectin immunoreactivity in neurons and dendrites, and a lack of staining in glial cells in the cerebrum and cerebellum, and the source of tetranectin in the CSF is believed to be the central nervous system [28]. Our previous study demonstrated that tetranectin was present at reduced levels in PD patients in comparison to normal controls [29]. In the present study, we further confirmed these results and found that tetranectin levels were significantly elevated after STN DBS. Since tetranectin is involved in the degradation of proteins in the brain [27], and accumulating evidence suggests that degeneration and loss of dopaminergic neurons in brain have links with abnormal protein aggregation [30-32], it would seem reasonable to assume that elevated tetranectin levels following STN DBS would reduce abnormal protein aggregation.

\section{Conclusion}

Using proteomic techniques and western blotting, we have studied for the first time proteome variations in the CSF of PD patients under pre- and post-DBS conditions in an attempt to elucidate the possible mechanisms of DBS. Our results revealed the presence of alterations in the levels of certain proteins in the CSF of PD patients during the perioperative and follow-up periods. Of these proteins, C4, apoA-I and IgA were stably expressed during the follow-up period while the levels of EC-SOD and tetranectin significantly increased. Our findings suggest that EC-SOD and tetranectin may serve as potential biomarkers of PD. But the exact roles of these two proteins in the brain still remain to be determined. Further validations of the results are required and should be performed in a larger cohort of DBS patients with a sufficient follow-up period.

\section{Abbreviations}

apoA-I (apolipoprotein A-I); CSF (cerebrospinal fluid); DBS (deep brain stimulation); EC-SOD (extracellular superoxide dismutase); H\&Y (Hoehn and Yahr); GPi (globus pallidus internus); LP (lumbar puncture); PD (Parkinson's disease); STN (subthalamic nucleus); UPDRS (Unified Parkinson's Disease Rating Scale); MSA (Multiple System Atrophy).

\section{Conflict of Interest}

The authors declare they have no conflict of interest. 


\section{Cellular Physiology \\ and Biochemistry}

Cell Physiol Biochem 2013;31:625-637

\begin{tabular}{l|l}
\hline DOI: $10.1159 / 000350082$ & (C) 2013 S. Karger AG, Basel
\end{tabular}

www.karger.com/cpb

Wang et al.: Proteomic Analysis of the Cerebrospinal Fluid of Parkinson's Disease Patients

\section{Acknowledgements}

This work was supported by grants from the Shanghai Municipal Commission for Science and Technology (No. 10140903500 and 124119a1900) and Shanghai Jiaotong University (No.YG2010MS40) and Shanghai Municipal Public Health Bureau (No.20124339).

\section{References}

1 Vitek J: Deep brain stimulation. J Clin Neurophysiol 2004;21:1.

2 Benabid AL, Wallace B, Mitrofanis J, Xia C, Piallat B, Fraix V, Batir A, Krack P, Pollak P, Berger F: Therapeutic electrical stimulation of the central nervous system. C R Biol 2005;328:177-186.

-3 Benabid AL, Koudsie A, Benazzouz A, Fraix V, Ashraf A, Le Bas JF, Chabardes S, Pollak P: Subthalamic stimulation for parkinson's disease. Arch Med Res 2000;31:282-289.

4 Lozano AM: Deep brain stimulation for parkinson's disease. Parkinsonism Relat Disord 2001;7:199-203.

5 Volkmann J: Deep brain stimulation for the treatment of parkinson's disease. J Clin Neurophysiol 2004;21:6-17.

6 McIntyre CC, Savasta M, Walter BL, Vitek JL: How does deep brain stimulation work? Present understanding and future questions. J Clin Neurophysiol 2004;21:40-50.

7 Kringelbach ML, Jenkinson N, Owen SL, Aziz TZ: Translational principles of deep brain stimulation. Nat Rev Neurosci 2007;8:623-635.

8 Benabid AL, Chabardes S, Mitrofanis J, Pollak P: Deep brain stimulation of the subthalamic nucleus for the treatment of parkinson's disease. Lancet Neurol 2009;8:67-81.

-9 Davidsson P, Sjogren M: The use of proteomics in biomarker discovery in neurodegenerative diseases. Dis Markers 2005;21:81-92.

10 Kim SI, Voshol H, van Oostrum J, Hastings TG, Cascio M, Glucksman MJ: Neuroproteomics: Expression profiling of the brain's proteomes in health and disease. Neurochem Res 2004;29:1317-1331.

-11 Ishigami N, Tokuda T, Ikegawa M, Komori M, Kasai T, Kondo T, Matsuyama Y, Nirasawa T, Thiele H, Tashiro K, Nakagawa M: Cerebrospinal fluid proteomic patterns discriminate parkinson's disease and multiple system atrophy. Mov Disord 2012;27:851-857.

12 Trojanowski JQ, Goedert M, Iwatsubo T, Lee VM: Fatal attractions: Abnormal protein aggregation and neuron death in parkinson's disease and lewy body dementia. Cell Death Differ 1998;5:832-837.

13 Paik SR, Lee D, Cho HJ, Lee EN, Chang CS: Oxidized glutathione stimulated the amyloid formation of alphasynuclein. FEBS Lett 2003;537:63-67.

14 Meredith GE, Halliday GM, Totterdell S: A critical review of the development and importance of proteinaceous aggregates in animal models of parkinson's disease: New insights into lewy body formation. Parkinsonism Relat Disord 2004;10:191-202.

15 McNaught KS, Olanow CW: Protein aggregation in the pathogenesis of familial and sporadic parkinson's disease. Neurobiol Aging 2006;27:530-545.

16 Davidsson P, Paulson L, Hesse C, Blennow K, Nilsson CL: Proteome studies of human cerebrospinal fluid and brain tissue using a preparative two-dimensional electrophoresis approach prior to mass spectrometry. Proteomics 2001;1:444-452.

17 Benabid AL: Deep brain stimulation for parkinson's disease. Curr Opin Neurobiol 2003;13:696-706.

18 Lang AE, Widner H: Deep brain stimulation for parkinson's disease: Patient selection and evaluation. Mov Disord 2002;17:S94-101.

19 Yoav B, Yosef H: On the adaptive control of the false discovery rate in multiple testing with independent statistics. J Behav Educ Statist 2000;25:60-83.

-20 Zech H, Echtermeyer C, Wohlbrand L, Blasius B, Rabus R: Biological versus technical variability in 2-d dige experiments with environmental bacteria. Proteomics 2011;11:3380-3389.

21 Alban A, David SO, Bjorkesten L, Andersson C, Sloge E, Lewis S, Currie I: A novel experimental design for comparative two-dimensional gel analysis: Two-dimensional difference gel electrophoresis incorporating a pooled internal standard. Proteomics 2003;3:36-44. 


\section{Cellular Physiology}

Cell Physiol Biochem 2013;31:625-637 and Biochemistry

\begin{tabular}{l|l}
\hline DOI: $10.1159 / 000350082$ & (c) 2013 S. Karger AG, Basel
\end{tabular}

Wang et al.: Proteomic Analysis of the Cerebrospinal Fluid of Parkinson's Disease Patients

-22 Keeney JT, Swomley AM, Förster S, Harris JL, Sultana R, Butterfield DA: Apolipoprotein a-i: Insights from redox proteomics for its role in neurodegeneration. Proteomics Clin Appl 2013;7:109-122.

23 Kroksveen AC, Opsahl JA, Aye TT, Ulvik RJ, Berven FS: Proteomics of human cerebrospinal fluid: Discovery and verification of biomarker candidates in neurodegenerative diseases using quantitative proteomics. J Proteomics 2011;74:371-388.

-24 Fattman CL, Schaefer LM, Oury TD: Extracellular superoxide dismutase in biology and medicine. Free Radic Biol Med 2003;35:236-256.

-25 Oury TD, Card JP, Klann E: Localization of extracellular superoxide dismutase in adult mouse brain. Brain Res 1999;850:96-103.

26 Obrist P, Spizzo G, Ensinger C, Fong D, Brunhuber T, Schafer G, Varga M, Margreiter R, Amberger A, Gastl G, Christiansen M: Aberrant tetranectin expression in human breast carcinomas as a predictor of survival. J Clin Pathol 2004;57:417-421.

-27 Dietzmann K, von Bossanyi P, Krause D, Wittig H, Mawrin C, Kirches E: Expression of the plasminogen activator system and the inhibitors pai-1 and pai-2 in posttraumatic lesions of the cns and brain injuries following dramatic circulatory arrests: An immunohistochemical study. Pathol Res Pract 2000;196:15-21.

28 Stoevring B, Jaliashvili I, Thougaard AV, Ensinger C, Hogdall CK, Rasmussen LS, Sellebjerg F, Christiansen M: Tetranectin in cerebrospinal fluid: Biochemical characterisation and evidence of intrathecal synthesis or selective uptake into csf. Clin Chim Acta 2005;359:65-71.

-29 Wang ES, Sun Y, Guo JG, Gao X, Hu JW, Zhou L, Hu J, Jiang CC: Tetranectin and apolipoprotein a-i in cerebrospinal fluid as potential biomarkers for parkinson's disease. Acta Neurol Scand 2010;122:350-359.

-30 Agorogiannis EI, Agorogiannis GI, Papadimitriou A, Hadjigeorgiou GM: Protein misfolding in neurodegenerative diseases. Neuropathol Appl Neurobiol 2004;30:215-224.

- 31 Ellisdon AM, Bottomley SP: The role of protein misfolding in the pathogenesis of human diseases. IUBMB Life 2004;56:119-123.

32 Gasser T: Genetics of parkinson's disease. Curr Opin Neurol 2005;18:363-369. 